BULL. AUSTRAL. MATH. SOC.

VOL. $10(1974), 147-148$.

\title{
Interior layers in rotating fluids
}

\section{Noel Geoffrey Barton}

A linear theory is used to investigate steady driven fluid motions in a rotating container whose bottom geometry forces the formation of interior layers. Essentially, this thesis contains three parts - derivation of linearized model equations for an incompressible fluid in any rotating container; calculation of the pressure field in a cylinder whose bottom is shaped as a parabolic trough; and consideration of a related singular perturbation problem posed by an elliptic equation with a small parameter.

The general theory, presented in Chapter 2, of the steady driven fluid motions in rotating containers of variable depth formally expresses the conservation of fluid in axial columns, and it re-presents known results in a form which permits the estimation of neglected terms. Formulae are derived for the fluid velocities in containers with closed and open axial depth function contours.

The steady driven motions in a rotating cylinder with a parabolic bottom are considered in Chapters 3 and 4 . Fluid speeds throughout most of the cylinder cross section are small, except for relatively high speeds in an interior layer where the axial depth function possesses a local maximum. Relatively large speeds also occur in two sidewall boundary layers which permit global mass conservation. The pressure at the line of maximum depth is given by a Fredholm integral equation of the first kind, which requires a well-conditioned numerical solution procedure.

The calculation of the interior layer pressure, using an approximating parabolic equation, is linked with a singular perturbation problem since the pressure is more accurately given by an elliptic equation whose highest derivatives have a small coefficient. A Sobolev-type inequality, derived

Received 28 June 1973. Thesis submitted to the University of Western Australia, June 1973. Degree approved, November 1973. Supervisor: Dr P. B. Chapman. 
in 55.3, is used to estimate the accuracy of the approximation, but it only leads to useful estimates in the interior layer. Known results guarantee the accuracy of the approximation elsewhere, apart from in a narrow transition region at the edge of the interior layer.

Finally, the construction of more accurate approximations, and the relation between the model problem and the physical situation. are examined in Chapter 6. 